\section{Relativistic Wave Equations}

Dirac's wave equation of a free electron may be expressed in the form

$$
\left(i \alpha_{\mu} \partial \mu+\chi\right) \psi=0, . \quad .
$$

where the matrices $\alpha_{\mu}(\mu=0,1,2,3)$ obey the commutation relations

$$
\alpha_{\mu} \alpha_{\nu}+\alpha_{\nu} \alpha_{\mu}=2 g_{\mu \nu} . . \quad .
$$

The usual notation is used, and $g_{\mu \nu}$ is the metric tensor for flat space-time given by $g_{00}=1, g_{11}=$ $g_{22}=g_{33}=-1$, the other components vanishing. The commutation relations (2) are such that from the first-order equation (1) one may deduce the secondorder equation

$$
\left(\partial_{\mu} \partial \mu+\chi^{2}\right) \psi=0 .
$$

In Kemmer's theory of the meson there are used certain matrices $\beta_{\mu}(\mu=0,1,2,3)$ which satisfy the commutation relations

$$
\beta_{\mu} \beta_{\nu} \beta_{\rho}+\beta_{\rho} \beta_{\nu} \beta_{\mu}=g_{\nu \rho} \beta_{\mu}+g_{\mu \nu} \beta_{\rho}, \quad .
$$

which ensure that the first-order equation

$$
\left(i \beta_{\mu} \partial \mu+\chi\right) \psi=0
$$

is consistent with the second-order equation (3).

The relations (4) are not symmetrical in all the suffixes, unlike in (2) where there is complete symmetry. Let us suppose that there exist $\varepsilon_{\mu}(\mu=0,1,2,3)$ which satisfy the symmetrical relations

$$
\begin{array}{r}
\left(\varepsilon_{\mu} \varepsilon_{\nu}+\varepsilon_{\nu} \varepsilon_{\mu}-2 g_{\mu \nu}\right) \varepsilon_{\rho}+\left(\varepsilon_{\nu} \varepsilon_{\rho}+\varepsilon_{\rho} \varepsilon_{\nu}-2 g_{\rho \nu}\right) \varepsilon_{\mu}+ \\
\left(\varepsilon_{\rho} \varepsilon_{\mu}+\varepsilon_{\mu} \varepsilon_{\rho}-2 g_{\mu \rho}\right) \varepsilon_{\nu}=0 .
\end{array}
$$

It is readily seen that $\alpha_{\mu}$ and $\beta_{\mu}$ both satisfy (5). That is, the matrices $\alpha_{\mu}$ and $\beta_{\mu}$ are possible representations of $\varepsilon_{\mu}$. It would be of interest to see if, apart from $\alpha_{\mu}$ and $\beta_{\mu}$, there exist other representations of $\varepsilon_{\mu}$, and if so whether they may be used to formulate a theory of particles. If we assume for a moment that a representation of $\varepsilon_{\mu}$ exists, we should then see whether the commutation relations satisfied by $\varepsilon_{\mu}$ are such that the first-order equation

$$
\left(i \varepsilon_{\mu} \partial \mu+\chi\right) \psi=0,
$$

leads to the second-order equation (3). It is easy to show that the commutation relations (5) satisfy this requirement. We operate on (6) from the right by $\left(\varepsilon_{v} \varepsilon_{\rho}+\varepsilon_{\rho} \varepsilon_{v}\right) \partial \nu \partial \rho$, and use (5). Then from (6) we obtain $\chi\left(\partial_{\mu} \partial \mu+\chi^{2}\right) \psi=0$, which is equivalent to $(3)$ when $\chi$ is not zero.

Now we come to the question of the representations of $\varepsilon_{\mu}$. So far as I am aware, the possible general representations of $\varepsilon_{\mu}$ given by (5) have not been investigated. It appears to me likely that the general relations (5) alone may not be sufficient to ensure a finite representation. More stringent requirements may be necessary. These extra conditions should be consistent with (5) and also should be relativistically invariant. Examples of such conditions are (2) and (4), which give the known representations $\alpha_{\mu}$ and $\beta_{\mu}$ respectively. It is not impossible that there may be other conditions which give new representations of $\varepsilon_{\mu}$ different from $\alpha_{\mu}$ and $\beta_{\mu}$.

This question is being investigated further and will be dealt with in a later communication.

Christ's College,

C. Jayaratnam Elifezer

Cambridge.

Nov. 4.

\section{Raman Spectrum of Diamond under High Resolution}

UsING the $\lambda 2536 \cdot 5$ radiation of the mercury arc as the exciter and a Hilger 'Intermediate' quartz spectrograph, the faint second-order Raman spectrum of diamond was first successfully recorded by $\mathrm{me}^{1}$. Later investigation $\mathbf{s}^{2,3}$ with somewhat larger diamonds and with a more powerful instrument, namely, a Hilger 'medium' quartz spectrograph, resulted in a notable improvement in the clarity of the recorded spectra. I have now succeeded in obtaining a satisfactorily exposed picture with a 'large' Hilger quartz spectrograph. The expectation that the greater dispersion and resolution given by this instrument would enable the true nature of the spectrum to be exhibited unambiguously has been fulfilled. Three prints of varying depth enlarged from the original spectrogram are reproduced herewith. The principal feature noticeable in the spectra is the appearance of a whole series of sharply defined Raman lines clearly resolved from each other. Twelve of the most prominent of these lines have been marked with their respective. frequency shifts in wave-numbers from the exciting radiation. The width of the individual Raman lines, as is evident from a comparison with the lines of the mercury arc, is no greater than that arising from the instrumental and photographic factors. Besides those marked, there are other fainter lines just discernible in the spectrogram against a fainter unresolved background of apparently continuous spectrum.

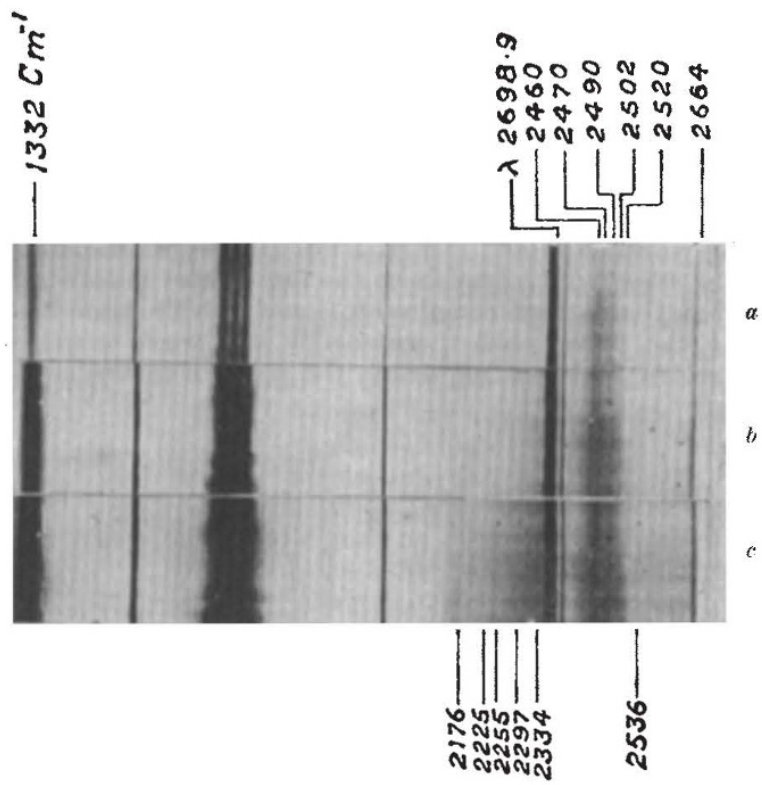

The experimental results shown in the spectra are obviously of great interest in relation to the fundamental problem of the nature of the vibration spectrum of a crystal lattice. The largest observed frequency shift in the second-order spectrum, namely, 2664 , is clearly the octave of 1332 appearing strongly in the first order. This suggests that all the other lines observed in the second-order Raman effect are likewise either octaves or else allowed combinations of discrete vibration frequencies of the crystal lattice which are Raman-inactive in the first order. This interpretation is supported by a large body of experimental evidence, for a detailed discussion of which reference should be made to some recent papers ${ }^{3,4,5}$. 